\title{
A DIRECT METHOD FOR SOLVING STOCHASTIC CONTROL PROBLEMS*
}

\author{
TYRONE E. DUNCAN ${ }^{\dagger}$ AND BOZENNA PASIK-DUNCAN ${ }^{\dagger}$
}

\begin{abstract}
In this paper a method motivated by completion of squares is used to describe explicit optimal controls for some stochastic control problems that include the linear-quadratic control problem for systems with a general noise process, the linear exponential quadratic Gaussian control problem for systems with Brownian motion, and the control of Brownian motion in the two sphere and the real hyperbolic plane with both finite and infinite time horizons.
\end{abstract}

1. Introduction. While the study of stochastic control problems can be traced from the evolution of the calculus of variations and deterministic control and from various stochastic control problems in statistics, the first major problem solution for the formally defined area of optimal stochastic control was the linear-quadratic Gaussian (LQG) problem (e.g. [2], [3]) that is also called the stochastic regulator problem (e.g. [13]). The area of stochastic control has developed significantly in breadth and depth from the solution of the LQG problem approximately fifty years ago. Two general methods have developed for solving stochastic control problems. They are the Hamilton-Jacobi-Belllman (HJB) equation and the stochastic maximum (or minimum) principle. The HJB equation can be considered as a natural generalization of the Hamilton-Jacobi equation of classical mechanics and the stochastic maximum principle can be considered as a natural generalization of the maximum principle of deterministic control (e.g. [18]).

For a stochastic control problem the Hamilton-Jacobi-Bellman equation is (typically) a nonlinear second order partial differential equation. The control system is assumed to generate a continuous time Markov process for any control from the family of admissible controls. Clearly the questions of existence and uniqueness of solutions for HJB equations are difficult in general. Therefore the notion of solutions is often relaxed to viscosity solutions [14]. However an optimal control that arises from the solution of an HJB equation may not be in the family of admissible controls. Thus significant difficulties often arise with this method though it provides sufficient conditions for optimality.

The stochastic maximum principle method is another important approach which yields necessary conditions for optimality. With some suitable convexity conditions

\footnotetext{
${ }^{*}$ Dedicated to Professor Hanfu Chen on the occasion of his 75th birthday. Research supported by NSF grants DMS 0808138 and DMS 1108884, AFOSR grants FA9550-09-12-1-0384 and FA9550-121-0384, and ARO grant W911NF-10-1-0248.

${ }^{\dagger}$ Department of Mathematics, University of Kansas, Lawrence, KS 66045.
} 
the necessary conditions of the stochastic maximum principle can be shown to be sufficient. In this approach a solution of a backward stochastic differential equation (BSDE) is required for the solution of the optimal control problem. A BSDE is solved backward in time but the solution is required to have a measurability that is forward in time. The questions of the existence and the uniqueness of solutions of BSDEs for stochastic control problems are generally difficult to resolve.

Both of these methods for the solutions of optimal stochastic control problems have formidable aspects. Another method that has been effectively used for linearquadratic deterministic control and LQG control arises from the completion of squares method in elementary algebra. This approach provides an optimal control directly. It is generalized here to other control problems. Some examples are provided to demonstrate the usefulness of this approach for problems of both linear and nonlinear stochastic systems, systems perturbed by general noise processes and infinite time horizon problems. While some results are only described, the proofs of some other results are either given or sketched.

Some advantages of this third direct method are that it is (mathematically) elementary, a general family of admissible controls can be considered, insight into the structure of the form of the explicit optimal controls can be obtained, and general noise processes other than Brownian motions can often be considered.

A brief outline of the paper is given now. In Section 2 control problems with a controlled linear system and either a general noise process and a quadratic cost functional or a Brownian motion with the exponential of a quadratic functional are explicitly solved. In Section 3 control problems for a Brownian motion in either a two sphere or a real hyperbolic plane are explicitly solved with both finite and infinite time horizons.

It is a pleasure for the authors to dedicate this paper to Han-Fu Chen on the occasion of his seventy-fifth birthday.

2. Control of Stochastic Linear Systems. Initially consider a linear-quadratic control problem described by the controlled linear system given by

$$
\begin{aligned}
d X(t) & =A X(t) d t+B U(t) d t+C d W(t) \\
X(0) & =X_{0}
\end{aligned}
$$

where $X_{0} \in \mathbb{R}^{n}$ is a constant vector, $X(t) \in \mathbb{R}^{n}, A \in \mathcal{L}\left(\mathbb{R}^{n}, \mathbb{R}^{n}\right), B \in \mathcal{L}\left(\mathbb{R}^{m}, \mathbb{R}^{n}\right), C \in$ $\mathcal{L}\left(\mathbb{R}^{p}, \mathbb{R}^{n}\right), U(t) \in \mathbb{R}^{m}, U \in \mathcal{U},(W(t), t \in[0, T])$ is an $\mathbb{R}^{p}$-valued square integrable process with continuous sample paths and this process is defined on the complete probability space $(\Omega, \mathcal{F}, \mathbb{P})$ and $T>0$ is fixed. Let $(\mathcal{F}(t), t \in[0, T])$ be the filtration of $(W(t), t \in[0, T])$. The family of adapted, admissible controls, $\mathcal{U}_{a}$, is 
$\mathcal{U}_{a}=\left\{U: U\right.$ is an $\mathbb{R}^{m}$-valued process that is progressively measurable with respect to $(\mathcal{F}(t), t \in[0, T])$ such that $U \in L^{2}([0, T])$ a.s. $\}$

The quadratic cost functional, $J$, for the control problem is given by

$$
\begin{aligned}
J^{0}(U)= & \frac{1}{2} \int_{0}^{T}(<Q X(s), X(s)>+<R U(s), U(s)>) d s \\
& +\frac{1}{2}<M X(T), X(T)> \\
J(U)= & \mathbb{E} J^{0}(U)
\end{aligned}
$$

where $Q \in \mathcal{L}\left(\mathbb{R}^{n}, \mathbb{R}^{n}\right), R \in \mathcal{L}\left(\mathbb{R}^{m}, \mathbb{R}^{m}\right), M \in \mathcal{L}\left(\mathbb{R}^{n}, \mathbb{R}^{n}\right), Q>0, R>0$ and $M \geq 0$ are symmetric linear transformations.

This linear-quadratic control problem can be explicitly solved as described in the following theorem. The result is a natural generalization of the result for Brownian motion by including in the optimal control a prediction of the response of the dual optimal system to the future behavior of the process $W$.

THEOREM 2.1. For the optimal control problem (1) and (3) and the family of admissible, adapted controls, $\mathcal{U}_{a}$, there is an optimal control $U^{*}$ that can be expressed as

$$
U^{*}(t)=-R^{-1} B^{T}(P(t) X(t)+V(t))
$$

where $(P(t), t \in[0, T])$ is the unique symmetric positive definite solution of the Riccati equation

$$
\begin{aligned}
\frac{d P}{d t} & =-P A-A^{T} P+P B R^{-1} B^{T} P-Q \\
P(T) & =M
\end{aligned}
$$

and $(V(t), t \in[0, T])$ is the process that satisfies

$$
V(t)=\mathbb{E}\left[\int_{t}^{T} \Phi_{P}(s, t) P(s) C d W(s) \mid \mathcal{F}(t)\right]
$$

and $\Phi_{P}$ is the fundamental solution of the matrix equation

$$
\begin{aligned}
\frac{d \Phi_{P}(s, t)}{d t} & =-\left(A^{T}-P(t) B R^{-1} B^{T}\right) \Phi_{P}(s, t) \\
\Phi_{P}(s, s) & =I .
\end{aligned}
$$

This result is proved in [9] and [11] by considering $W$ as an affine term of the equation (1), generalizing some methods for affine terms of deterministic linear control systems, and using conditional expectation.

An important variation of the linear-quadratic Gaussian control problem is the linear exponential quadratic Gaussian problem where the control system is the same 
as in (1) and $W$ is a standard Brownian motion but the cost functional is changed to the exponential of a quadratic functional, that is, $J^{0}$ in (3) is replaced by

$$
\begin{aligned}
J_{e}^{0}(U) & =\exp \left[\frac{\mu}{2} \int_{0}^{T}(<Q X(t), X(t)>+<R U(t), U(t)>) d t\right. \\
& \left.+\frac{\mu}{2}<M X(T), X(T)>\right] \\
J_{e}(U) & =\mathbb{E}\left[J_{e}^{0}(U)\right]
\end{aligned}
$$

where $\mu>0$ is fixed and suitably bounded. This problem is an example of a risk sensitive control problem (e.g. [12]) and in economic terms $\mu>0$ describes an investor who is risk averse. This problem is well posed for $\mu \in\left(0, \mu_{0}\right)$ where $\mu_{0}$ is determined from the solution region of a Riccati equation associated with this control problem.

The family of admissible controls, $\mathcal{U}$, is

$\mathcal{U}=\left\{U: U\right.$ is an $\mathbb{R}^{m}$-valued process that is progressively measurable with respect to $(\mathcal{F}(t), t \in[0, T])$ such that $U \in L^{2}([0, T])$ a.s. $\}$

While Jacobson [17] proved this result by explicitly solving the associated Hamilton-Jacobi-Bellman equation, the proof outlined here is more direct and additionally provides an explanation for the difference between the Riccati equation for this solution and the Riccati equation for the LQG solution. The following result provides an optimal control. A sketch of the proof is also given and a complete proof is given in [8].

TheOREM 2.2. For the control problem given by (1) and (12) where $W$ in (1) is a standard Brownian motion, there is an optimal control $\left(U^{*}(t), t \in[0, T]\right)$ in $\mathcal{U}$ given by

$$
U^{*}(t)=-R^{-1} B^{T} P(t) X(t)
$$

where $(P(t), t \in[0, T])$ is assumed to be the unique, symmetric, positive solution of the following Riccati equation

$$
\begin{aligned}
& -\frac{d P}{d t}=P A+A^{T} P-P\left(B R^{-1} B^{T}-\mu C C^{T}\right) P+Q \\
& P(T)=M
\end{aligned}
$$

and the optimal cost is

$$
J\left(U^{*}\right)=G(0) \exp \left[\frac{\mu}{2}<P(0) X_{0}, X_{0}>\right]
$$

and $(G(t), t \in[0, T])$ satisfies

$$
\begin{aligned}
-\frac{d G}{d t} & =\frac{\mu}{2} G \operatorname{tr}\left(P C C^{T}\right) \\
G(T) & =1 .
\end{aligned}
$$


Proof. (Sketch). The cost functional $J_{e}$ is written as

$$
J_{e}(U)=\mathbb{E} \exp [K(U)]
$$

and $K(U)$ can be expressed in the following form using the change of variables formula for stochastic differential equations applied to $\langle P(t) X(t), X(t)\rangle, t \in[0, T])$ where $P$ is the solution of (14)

$$
\begin{aligned}
& K(U)-\frac{\mu}{2}<P(0) X_{0}, X_{0}> \\
& =\frac{\mu}{2}\left[\int_{0}^{T}\left(<R U, U>+<P B R^{-1} B^{T} P X, X>+2<B^{T} P X, U>\right) d t\right. \\
& \left.+2 \int_{0}^{T}<P X, C d W>-\mu \int_{0}^{T}<P C C^{T} P X, X>d t+\int_{0}^{T} \operatorname{tr}\left(P C C^{T}\right) d t\right] \\
& =\frac{\mu}{2} \int_{0}^{T}\left|R^{-\frac{1}{2}}\left[R U+B^{T} P X\right]\right|^{2} d t \\
& +\mu \int_{0}^{T}<P X, C d W>-\frac{\mu^{2}}{2} \int_{0}^{T}<P C C^{T} P X, X>d t \\
& +\frac{\mu}{2} \int_{0}^{T} \operatorname{tr}\left(P C C^{T}\right) d t .
\end{aligned}
$$

The exponential of the two integrals that are the second and the third on the RHS of the last equality in (17) is a Radon-Nikodym derivative so the optimal control is obtained by minimizing the first term on the RHS of the last equality. This equality shows a crucial difference between the quadratic cost functionals and the exponential of the quadratic cost functionals and provides an explanation why the Riccati equation has to be modified for the exponential of a quadratic cost functional as compared to the quadratic cost functional. The optimal cost is obtained from the last term on the RHS of (17).

3. Control of Brownian Motion in Some Manifolds. This direct method that is demonstrated in Section 2 for solving control problems for linear systems and is derived from the completion of squares method is not restricted to linear control systems. The following examples of control of a Brownian motion in $S^{2}$, a two-sphere, and in $\mathbb{H}^{2}(\mathbb{R})$, a real hyperbolic plane, demonstrate the method's applicability to control problems for nonlinear systems.

The sphere $S^{2}$ is diffeomorphic to the rank one symmetric space $S O(3) / S O(2)$ and is a simply connected compact Riemannian manifold of constant positive sectional curvature (e.g. [15]). A Riemannian metric for $S^{2}$ is obtained by restricting the standard metric in $\mathbb{R}^{3}$. The maximal distance between any two points in $S^{2}$ using this metric is $L$ where

$$
L=\pi
$$


$L$ is called the diameter of $S^{2}$. Choose an origin $o \in S^{2}$. The antipodal point of $o$ is the submanifold $A_{o}$ that is the distance $L$ from $o$. Let $T_{o} S^{2}$ be the tangent space to $S^{2}$ at $o$. The exponential mapping $\exp _{o}: T_{o} S^{2} \rightarrow S^{2}$ is a diffeomorphism of the open ball $B_{L}(o)=\left\{x \in T_{o} S^{2}:|x|<L\right\}$ onto the open set $S^{2} \backslash A_{o}$. This diffeomorphism is explicitly given by the geodesic polar coordinates for $S^{2}$ at the origin as the map

$$
\exp _{o} Y \rightarrow(r, \theta)
$$

where $Y \in B_{L}(o), r=|Y|$ and $\theta$ is the local coordinate of the unit vector $Y /|Y|$. In these coordinates the Laplace-Beltrami operator $\Delta_{S^{2}}$ (e.g. p.169 [15]) is

$$
\Delta_{S^{2}}=\frac{\partial^{2}}{\partial r^{2}}+\cot \left(\frac{r}{2}\right) \frac{\partial}{\partial r}+\Delta_{S_{r}}
$$

where $r \in(0, L)$ and $\Delta_{S_{r}}$ is the Laplace-Beltrami operator on the sphere of radius $r$ from the origin. The sum of the first two terms on the right hand side of (20) is called the radial part of the Laplace-Beltrami operator. For the control problem here it is not necessary to describe $\Delta_{S_{r}}$. The controlled stochastic system is described by the following equation which describes the distance of the controlled Brownian motion from $o$.

$$
\begin{aligned}
d X(t) & =\frac{1}{2} \cot \left(\frac{X(t)}{2}\right) d t+U(t) d t+d B(t) \\
X(0) & =X_{0}
\end{aligned}
$$

where $X(t)$ is the radial distance, $(B(t), t \in[0, T])$ is a real-valued standard Brownian motion for a fixed $T>0$, and $X_{0} \in(0, L)$ is a constant. The Brownian motion is defined on the complete probability space $(\Omega, \mathcal{F}, \mathbb{P})$ and $(\mathcal{F}(t), t \in[0, T])$ is the filtration for the Brownian motion $B$. If $U(t)$ is a smooth function of $X(t)$ then $(X(t), t \in[0, T])$ is a Markov process with the infinitesimal generator

$$
\frac{1}{2} \frac{\partial^{2}}{\partial r^{2}}+\frac{1}{2} \cot \left(\frac{r}{2}\right) \frac{\partial}{\partial r}+U(r) \frac{\partial}{\partial r} .
$$

The cost functional for the control problem is denoted $J_{S}(U)$ that is described as follows

$$
\begin{aligned}
& J_{S}^{0}(U)=\int_{0}^{T}\left(a \sin ^{2} \frac{X(t)}{4}+U^{2}(t) \cos ^{2} \frac{X(t)}{4}\right) d t \\
& J_{S}(U)=\mathbb{E} J_{S}^{0}(U)
\end{aligned}
$$

where $a>0$ is a constant. The cost functional only depends on the radial distance from the origin $o$, so the control only appears in the radial component of the process. Note that $\sin ^{2} \frac{x}{4}$ is an increasing function for $x \in(0, \pi)$. The family of admissible controls, $\mathcal{U}$, is 
$\mathcal{U}=\{U \mid U:[0, T] \times \Omega \rightarrow \mathbb{R}$ is jointly measurable, $(U(t), t \in[0, T])$

is progressively measurable with respect to $(\mathcal{F}(t), t \in[0, T])$ and $\int_{0}^{T}|U(t)|^{2} d t<\infty$ a.s. $\}$

This family of controls is a natural family of processes in the equation (21) to ensure that the solution of the stochastic equation is well defined. The following Riccati and linear equations are used in the solution of the control problem.

$$
\begin{aligned}
\frac{d g(t)}{d t} & =\frac{3}{8} g+\frac{1}{16} g^{2}-a \\
g(T) & =0 \\
\frac{d h(t)}{d t} & =-\frac{3}{16} g \\
h(T) & =0 .
\end{aligned}
$$

The following result describes an optimal control for the stochastic system (21) and the cost functional (25) [10].

TheOREM 3.1. The stochastic control problem described by (21) and (25) has an optimal admissible control, $U^{*}$, that is given by

$$
U^{*}(t)=-\frac{1}{4} g(t) \tan \frac{X(t)}{4}
$$

where $t \in[0, T]$ and $g$ satisfies (26). The optimal cost is

$$
J\left(U^{*}\right)=g(0) \sin ^{2} \frac{X(0)}{4}+h(0)
$$

where $h$ satisfies (28).

The optimal control is obtained by applying the change of variables formula for stochastic differentials to $(Z(t), t \in[0, T])$ where $f(t, x)=g(t) \sin ^{2} \frac{x}{4}+h(t)$ and $Z(t)=$ $f(t, X(t))([10])$.

A noncompact space is now considered for a stochastic control problem. A real hyperbolic two space, $\mathbb{H}^{2}(\mathbb{R})$, is a noncompact symmetric space of rank one that can be expressed as a quotient of semisimple Lie groups as $G / K=S L(2, \mathbb{R}) / S O(2)$. While various geometric models exist for $\mathbb{H}^{2}(\mathbb{R})$, the unit disk model, $B_{1}(0)=\{y=$ $\left.\left(y_{1}, y_{2}\right) \in \mathbb{R}^{2}:|y|<1\right\}$ where $|\cdot|$ is the usual Riemannian metric in $\mathbb{R}^{2}$ is particularly convenient here. The unit disk is given the Riemannian structure

$$
d s^{2}=4\left(1-|y|^{2}\right)^{-2}\left(d y_{1}^{2}+d y_{2}^{2}\right) .
$$

The radial part, $R(\cdot)$, of the Laplace-Beltrami operator or equivalently the Laplace-Beltrami operator acting on $\mathrm{SO}(2)$-invariant functions is given by

$$
R\left(\Delta_{G / K}\right)=\frac{\partial^{2}}{\partial r^{2}}+\operatorname{coth} \frac{r}{2} \frac{\partial}{\partial r} .
$$


The controlled stochastic system for the distance from the origin $o$ is

$$
\begin{aligned}
d X(t) & =\frac{1}{2} \operatorname{coth} \frac{X(t)}{2} d t+U(t) d t+d B(t) \\
X(0) & =X_{0}
\end{aligned}
$$

The Brownian motion is defined on the complete probability space $(\Omega, \mathcal{F}, \mathbb{P})$ and $(\mathcal{F}(t), t \in[0, T])$ is the filtration for the Brownian motion $B$. If $U \equiv 0$ then the Markov process $X$ has infinitesimal generator $R\left(\Delta_{G / K}\right)$, the radial part of the Laplacian on $\mathbb{H}^{2}(\mathbb{R})$. The cost functional $J_{H}(U)$ is described as follows

$$
\begin{aligned}
& J_{H}^{0}(U)=\int_{0}^{T}\left(a \sinh ^{2} \frac{X(t)}{4}+U^{2}(t) \cosh ^{2} \frac{X(t)}{4}\right) d t \\
& J_{H}(U)=\mathbb{E} J_{H}^{0}(U)
\end{aligned}
$$

where $a>0$ is fixed. The family of admissible controls, $\mathcal{U}$, is

$\mathcal{U}=\{U \mid U:[0, T] \times \Omega \rightarrow \mathbb{R}$ is jointly measurable, $(U(t), t \in[0, T])$

is progressively measurable with respect to $(\mathcal{F}(t), t \in[0, T])$ and $\int_{0}^{T}|U(t)|^{2} d t<\infty$ a.s. $\}$

The following Riccati equation and linear equation are used to determine an optimal control.

$$
\begin{aligned}
\frac{d g}{d t} & =-\frac{3}{8} g+\frac{1}{16} g^{2}-a \\
g(T) & =0 \\
\frac{d h}{d t} & =-\frac{3}{16} g \\
h(T) & =0 .
\end{aligned}
$$

The following theorem describes an optimal control for the stochastic system (34) and the cost functional (37).

THEOREM 3.2. The stochastic control problem described by (34) and (37) has an optimal admissible control, $U^{*}$, that is given by

$$
U^{*}(t)=-\frac{1}{4} g(t) \tanh \frac{X(t)}{4}
$$

where $g$ satisfies (38). The optimal cost is

$$
J_{H}\left(U^{*}\right)=g(0) \sinh ^{2} \frac{X(0)}{4}+h(0)
$$

where $h$ satisfies (40). 
Proof. The following two well known hyperbolic sine and cosine identities are used below.

$$
\begin{aligned}
& \sinh ^{2} \frac{r}{2}=\frac{1}{2}(\cosh r-1) \\
& \frac{1}{2} \sinh r=\sinh \frac{r}{2} \cosh \frac{r}{2} .
\end{aligned}
$$

Let $f(t, x)=g(t) \sinh ^{2} \frac{x}{4}+h(t)$ and $Y(t)=f(t, X(t))$. Apply the stochastic differential rule (change of variables) to the process $(Y(t), t \in[0, T])$ and integrate this differential on $[0, T]$ to obtain

$$
\begin{gathered}
Y(T)-Y(0)=\int_{0}^{T}\left(\frac { g } { 2 } \operatorname { s i n h } \frac { X } { 4 } \operatorname { c o s h } \frac { X } { 4 } \left(\frac{1}{2} \operatorname{coth} \frac{X}{2} d t\right.\right. \\
+U d t+d B(t))+\frac{g}{16} \cosh \frac{X}{2} d t-\frac{3}{16} g d t \\
\left.+\left[-\frac{3 g}{8}+\frac{1}{16} g^{2}-a\right] \sinh ^{2} \frac{X}{4} d t\right) \\
=\int_{0}^{T}\left(\frac{g}{8} \cosh \frac{X}{2} d t+\frac{g}{2} U \sinh \frac{X}{4} \cosh \frac{X}{4} d t\right. \\
+\frac{g}{4} \sinh \frac{X}{2} d B(t)+\frac{g}{16} \cosh \frac{X}{2} d t-\frac{3}{16} g d t \\
\left.-\frac{3 g}{8} \frac{1}{2}\left(\cosh \frac{X}{2}-1\right) d t+\left(\frac{g^{2}}{16}-a\right) \sinh \frac{X}{4} d t\right) \\
=\int_{0}^{T}\left(\frac{g}{2} U \sinh \frac{X}{4} \cosh \frac{X}{4} d t+\frac{g}{4} \sinh \frac{X}{2} d B(t)\right. \\
\left.+\left(\frac{g^{2}}{16}-a\right) \sinh ^{2} \frac{X}{4} d t\right) .
\end{gathered}
$$

Using (36) the following equality is satisfied.

$$
\begin{aligned}
J_{H}^{0}(U) & -g(0) \sinh ^{2} \frac{X(0)}{4}-h(0) \\
& =\int_{0}^{T}\left(U^{2} \cosh ^{2} \frac{X}{4} d t+\frac{g}{2} U \sinh \frac{X}{4} \cosh \frac{X}{4} d t\right. \\
& \left.+\frac{g^{2}}{16} \sinh ^{2} \frac{X}{4} d t+\frac{g}{4} \sinh \frac{X}{2} d B(t)\right) \\
& =\int_{0}^{T}\left(\cosh ^{2} \frac{X}{4}\left(U^{2}+\frac{g}{2} U \tanh \frac{X}{4}+\frac{g^{2}}{16} \tanh ^{2} \frac{X}{4}\right) d t\right. \\
& \left.+\frac{g}{4} \sinh ^{\frac{X}{2}} d B(t)\right) \\
& =\int_{0}^{T}\left(\cosh ^{2} \frac{X}{4}\left(U+\frac{g}{4} \tanh \frac{X}{4}\right)^{2} d t+\frac{g}{4} \sinh \frac{X}{2} d B(t)\right) .
\end{aligned}
$$

It can be shown e.g. [7] that

$$
\mathbb{E} \int_{0}^{T} g^{2} \sinh ^{2} \frac{X}{2} d t<\infty
$$


Taking the expectation of (47) it is clear that an optimal control is

$$
U^{*}(t)=-\frac{1}{4} g(t) \tanh \frac{X(t)}{4} .
$$

The optimal cost is

$$
J_{H}\left(U^{*}\right)=g(0) \sinh ^{2} \frac{X(0)}{4}+h(0) .
$$

It should be noted the similarity or more precisely the duality between the optimal controls and the optimal costs in the last two theorems for stochastic systems in $S^{2}$ and $\mathbb{H}^{2}(\mathbb{R})$ respectively. This duality is a generalization of the duality from Lie theory between the compact symmetric space $S^{2}$ and the noncompact symmetric space $\mathbb{H}^{2}(\mathbb{R})$.

As a final example of this direct approach, an infinite time horizon discounted control problem for this same control system in $\mathbb{H}^{2}(\mathbb{R}),(34)$, is solved. Let $\rho>0$ be fixed. The cost functional, $J_{\rho}$, is

$$
\begin{aligned}
& J_{\rho}^{0}(U)=\int_{0}^{\infty} e^{-\rho t}\left(a \sinh ^{2} \frac{X(t)}{4}+U^{2}(t) \cosh ^{2} \frac{X(t)}{4}\right) d t \\
& J_{\rho}(U)=\mathbb{E} J_{\rho}^{0}(U) .
\end{aligned}
$$

The family of admissible controls, $\mathcal{U}_{\rho}$, is

$\mathcal{U}_{\rho}=\{U \mid U:[0, \infty) \times \Omega \rightarrow \mathbb{R}$ is jointly measurable, $(U(t), t \in[0, \infty))$

is progressively measurable with respect to $(\mathcal{F}(t), t \in[0, \infty))$ and $\int_{0}^{T}|U(t)|^{2} 1_{[0, T]} d t<\infty$ a.s. for each $\left.T>0.\right\}$

The following algebraic Riccati equation and linear differential equation are used in the determination of the optimal control. The positive root of the Riccati equation is chosen.

$$
\begin{aligned}
& \frac{1}{16} g^{2}-g\left(-\rho+\frac{3}{8}\right)-a=0 \\
& \frac{d h}{d t}=-\frac{3}{16} g e^{-\rho t} \\
& h(0)=0 .
\end{aligned}
$$

The following theorem solves the stochastic control problem by providing explicitly an optimal control and the optimal cost.

TheOREM 3.3. The stochastic control problem, described by (34) and (52), has an optimal admissible control, $U_{\rho}^{*}$, that is given by

$$
U_{\rho}^{*}=-\frac{g}{4} \tanh \frac{X(t)}{4}
$$


where $g$ is the positive root of (53). The optimal cost is

$$
J_{\rho}\left(U_{\rho}^{*}\right)=g \sinh ^{2} \frac{X(0)}{4}-h(\infty)
$$

Proof. Let $\rho>0$ be given in the cost functional, (52), and let

$$
f(t, x)=g e^{-\rho t} \sinh ^{2} \frac{x}{4}+h(t)
$$

where $h$ satisfies (54). Let $Y(t)=f(t, X(t))$ where $X$ satisfies (34) and apply the change of variables formula to $(Y(t), t \in[0, T])$ for a $T>0$ fixed.

$$
\begin{array}{r}
Y(T)-Y(0)=\int_{0}^{T} \frac{g}{2} e^{-\rho t} \sinh \frac{X}{4} \cosh \frac{X}{4}\left(\frac{1}{2} \operatorname{coth} \frac{X}{2} d t+U d t+d B\right) \\
+\frac{g}{16} e^{-\rho t} \cosh \frac{X}{2} d t-\rho g e^{-\rho t} \sinh ^{2} \frac{X}{4} d t-\frac{3}{16} g e^{-\rho t} d t \\
=\int_{0}^{T}\left(\frac{g}{8} e^{-\rho t} \cosh \frac{X}{2} d t+\frac{g}{2} e^{-\rho t} U \sinh \frac{X}{4} \cosh \frac{X}{4} d t\right. \\
+\frac{g}{4} e^{-\rho t} \sinh \frac{X}{2} d B+\frac{g}{16} e^{-\rho t} \cosh \frac{X}{2} d t \\
\left.-\rho g e^{-\rho t} \sinh ^{2} \frac{X}{4} d t-\frac{3}{16} g e^{-\rho t} d t\right) \\
=\int_{0}^{T}\left(\frac{g}{8} e^{-\rho t}\left(2 \sinh \frac{X}{4}+1\right) d t+\frac{g}{2} e^{-\rho t} U \sinh \frac{X}{4} \cosh \frac{X}{4} d t\right. \\
+\frac{g}{4} e^{-\rho t} \sinh \frac{X}{2} d B+\frac{g}{16} e^{-\rho t}\left(2 \sinh \frac{X}{4}+1\right) d t \\
\left.-\rho g e^{-\rho t} \sinh ^{2} \frac{X}{4} d t-\frac{3}{16} g e^{-\rho t} d t\right) .
\end{array}
$$

Using the equation for $g$ and the definition of the cost functional, (52), the following equality can be verified.

$$
\begin{array}{r}
\int_{0}^{T} e^{-\rho t}\left(a \sinh ^{2} \frac{X(t)}{4}+U^{2}(t) \cosh ^{2} \frac{X(t)}{4}\right) d t \\
+g e^{-\rho T} \sinh ^{2} \frac{X(T)}{4}-g \sinh ^{2} \frac{X(0)}{4}+h(T) \\
=\int_{0}^{T}\left(U^{2} e^{-\rho t} \cosh ^{2} \frac{X}{4}+\frac{g}{2} e^{-\rho t} U \sinh \frac{X}{4} \cosh \frac{X}{4}\right. \\
\left.+\frac{1}{16} g^{2} e^{-\rho t} \sinh ^{2} \frac{X}{4}\right) d t+\frac{g}{4} e^{-\rho t} \sinh \frac{X}{2} d B \\
=\int_{0}^{T}\left(e ^ { - \rho t } \operatorname { c o s h } \frac { X } { 4 } \left(U^{2}+\frac{g}{2} U \tanh \frac{X}{4}\right.\right. \\
\left.\left.+\frac{g^{2}}{16} \tanh ^{2} \frac{X}{4}\right) d t+\frac{g}{4} e^{-\rho t} \sinh \frac{X}{4} d B\right) \\
=\int_{0}^{T}\left(e^{-\rho t} \cosh ^{2} \frac{X}{4}\left(U+\frac{g}{4} \tanh ^{2}\right)^{2} d t+\frac{g}{4} e^{-\rho t} \sinh \frac{X}{2} d B\right) .
\end{array}
$$


Letting $U$ be the optimal control $U_{\rho}^{*}$, using an integrability property as in (48) and letting $T \rightarrow \infty$ by the Monotone Convergence Theorem it suffices to verify that

$$
\lim \sup _{T \rightarrow \infty} \mathbb{E} e^{-\rho T} \sinh ^{2} \frac{X(T)}{4}=0 .
$$

This result follows by a using a comparison theorem for scalar stochastic differential equations [16] where the optimal system equation is compared to

$$
\begin{aligned}
d Y(t) & =\frac{1}{2} \operatorname{coth} \frac{Y(t)}{2} d t-\frac{\alpha g}{2} d t+d B(t) \\
Y(t) & =X(0)
\end{aligned}
$$

for an $\alpha<1$ that can be chosen arbitrarily close to 1 and fixed. Thus the optimal control is $U_{\rho}^{*}$.

The optimal cost can be easily computed from the determination of $U_{\rho}^{*}$.

The method described in this paper provides an alternative to either the Hamilton-Jacobi-Bellman equation method or the stochastic maximum principle method. The method given here exhibits an optimal control and the optimal cost directly in the problem solution.

\section{REFERENCES}

[1] B. D. O Anderson and J. B. Moore, Linear Optimal Control, Prentice-Hall, New Jersey, 1971.

[2] H. F. Chen And L. Guo, Identification and Stochastic Adaptive Control, Birkhauser-Springer, New York, 1991.

[3] H. F. Chen, T. E. Duncan, And B. Pasik-Duncan, Stochastic adaptive control for continuous time linear systems with quadratic cost, Appl. Math. Optim., 34(1996), pp. 113-138.

[4] T. E. Duncan, A solvable stochastic control problem in hyperbolic three space, Systems Control Lett., 8(1987), pp. 435-439.

[5] T. E. Duncan, A solvable stochastic control problem in spheres, Contemp. Math. 73(1988), pp. 49-54.

[6] T. E. Duncan, A solvable stochastic control problem in the hyperbolic plane, J. Math. Systems, Estimation, Control, 2(1992), pp. 445-452.

[7] T. E. Duncan, Some solvable infinite time horizon stochastic control problems in hyperbolic three space, Proc. Symp. Pute Math., 64(1999), pp. 199-206.

[8] T. E. Duncan, Linear exponential quadratic Gaussian control, IEEE Trans. Autom. Control, accepted for publication.

[9] T. E. Duncan And B. Pasik-Duncan, Stochastic linear-quadratic control for systems with a fractional Brownian motion, Proc.49th IEEE Conference on Decision and Control, Atlanta, 2010, 6163-6168.

[10] T. E. Duncan and B. Pasik-Duncan, A control problem in the two-sphere, Proc. IEEE Multiconf. Systems Control, 2012, Dubrovnik, 1441-1444.

[11] T. E. Duncan And B. Pasik-Duncan, Linear quadratic fractional Gaussian control, preprint.

[12] W. F. Fleming and W. M. McEneaney, Risk-sensitive control on an infinite horizon, SIAM J. Control Optim. 33 (1995), pp. 1881-1915.

[13] W. H. Fleming and R. W. Rishel, Deterministic and Stochastic Optimal Control, Springer, New York, 1975. 
[14] W. H. Fleming and H. M. Soner, Controlled Markov Process and Viscosity Solutions, Springer, New York, 1993.

[15] S. Helgason, Groups and Geometric Analysis, Academic Press, New York, 1984.

[16] N. Ikeda and S. Watanabe, Stochastic Differential Equations and Diffusion Processes, North Holland, 1989.

[17] D. H. JACoBson, Optimal stochastic linear systems with exponential performance criteria and their relation to deterministic differential games, IEEE Trans. Autom. Control AC18 (1973), pp. 124-131.

[18] L. S. Pontryagin, V. G. Boltyanski, R. V. Gamkrelidze, and E. F. Mischenko, Mathematical Theory of Optimal Processes, Wiley, New York 1962. 
